\title{
Editorial
}

\section{Participants with Treated Hyperlipidemia may be More Health Conscious}

\author{
Atsushi Hozawa $^{1}$ and Tomonori Okamura ${ }^{2}$
}

${ }^{1}$ Division of Personalized Prevention and Epidemiology, Tohoku Medical Megabank Organization, Tohoku University, Sendai, Japan ${ }^{2}$ Department of Preventive Medicine and Public Health, School of Medicine, Keio University, Tokyo, Japan

\section{See article vol. 22: 1030-1039}

Hyperlipidemia is a well-known risk factor of coronary heart diseases, and the beneficial effect of hyperlipidemia treatment on coronary disease prevention was well established ${ }^{1-3)}$. Actually, US preventive service task force strongly recommends the screening of high blood cholesterol and its early treatment ${ }^{4)}$. However, to interpret the relationship of total cholesterol with all-cause mortality, several issues to be discussed were previously raised ${ }^{5,6}$. A report by Daimon, et al. contains these issues ${ }^{7)}$. In this editorial, we would like to introduce these topics.

Daimon et al. reported the relation of treated hyperlipidemia, untreated hyperlipidemia, and nonhyperlipidemia participants with total mortality ${ }^{7)}$. They concluded that participants with untreated hyperlipidemia showed a higher risk of total mortality than those with treated hyperlipidemia.

Their observation that treated hyperlipidemia participants showed a lower risk than untreated hyperlipidemia participants was in line with previous $\mathrm{RCTs}^{1-3)}$. It is confirmative that participants with hypercholesterolemia can improve their prognosis using lipid lowering medications just as clearly indicated in clinical guidelines worldwide ${ }^{1-3)}$

However, present finding was too striking, i.e., hazard ratio $(95 \%$ confidence interval) of participants with treated hyperlipidemia was 0.24 (0.08-0.69) compared with those with untreated hyperlipidemia, which may mislead readers concerned about the real effects of medication for dyslipidemia. This specious large difference cannot be explained only by the effects

Address for correspondence: Atsushi Hozawa, Division of Personalized Prevention and Epidemiology, Tohoku Medical Megabank Organization, Tohoku University, 2-1, Seiryo-machi,

Aoba-ku, Sendai, Miyagi 980-8575, Japan

E-mail: hozawa-thk@umin.ac.jp

Received: June 4, 2015

Accepted for publication: June 4, 2015 of lipid-lowering drugs; this difference can also be explained by the better health consciousness in treated hyperlipidemia participants.

Regarding hypertension, a previous study reported that only $24 \%$ of the untreated hypertensive participants who pointed out their high blood pressure at health check-up started treatment within 1 year ${ }^{8)}$. Because Tanaka et al. reported that the percentage of untreated subjects with hypertension was significantly lower than the percentage of untreated subjects with hypercholesterolemia ( $41.0 \%$ vs. $57.1 \%, p<$ $0.001)$ in Japanese workers, the rate of starting lipid medication may be lower than that of starting antihypertensive medication ${ }^{9)}$. These finding suggested that participants who treated hyperlipidemia were highly health conscious, which related to a healthy life style and good compliance for lifestyle modifications as advised by physicians. In the present study, Daimon et al. also revealed that the proportion of participants who were taking lipid lowering agents was only around 20\% (313/1395) in all dyslipidemic participants. Higher health consciousness in participants with treated hyperlipidemia was also suggested by the prevalence of current smoker, which is an indicator of healthy lifestyle. Prevalence of current smoker was $9.3 \%, 20.5 \%$, and $19.6 \%$ in participants with treated hyperlipidemia, untreated hyperlipidemia, and nonhyperlipidemia, respectively.

Thus, we could interpret the present findings that participants with treated hyperlipidemia showed a much lowest risk of total mortality not only because of the effect of lipid lowering medication but also because of higher health consciousness, in other words, a healthy lifestyle, their original personality,etc.

On the other hand, this paper revealed that crude mortality rate was lower in participants with hyperlipidemia without medication than in non-hyperlipidemic participants. Of course, this odd finding was diminished when they adjusted for possible confounders. However, the point estimate of risk was still lower in untreated hyperlipidemia than that in non-hyper- 
lipidemia participants. In previous studies, these findings that lower cholesterol was related to a higher mortality, particularly in non-coronary disease mortality, were observed. This finding may be mainly explained by subclinical exhausting condition, such as cancer, respiratory function, etc. For example, Okamura et al. investigated the relationship between total cholesterol and all-cause mortality ${ }^{10)}$. In their report, the highest cholesterol category as well as the lowest cholesterol group showed a higher risk of all-cause mortality. This relationship was not found after excluding deaths in the first 5 years of follow-up, i.e., in the group with lower cholesterol, the proportion of exhausted condition, such as liver diseases, depression may be increased.

Another interpretation was unmeasured confounding. Serum cholesterol level was defined mainly by diet, particularly for saturated fat intake. This westernized dietary style may be the marker of participants' higher socio-economic status (SES), i.e., higher saturated fatty acid intake related to the high serum level of cholesterol may be associated with higher SES, particularly in the rural population. This may also partly explain the higher mortality in lower cholesterol groups. In any case, it is cautious to interpret the observational data. Careful discussion including biological mechanisms is required.

The study reported by Daimon et al, raises several interesting points of discussion as shown above. The most important message may be that hypercholesterolemia participants with treatment show very low mortality. Together with the effect of lipid lowering agents, their better health consciousness may extend their life expectancies.

\section{Conflicts of Interest}

None.

\section{References}

1) Stone NJ, Robinson JG, Lichtenstein AH, Bairey Merz CN, Blum CB, Eckel RH, Goldberg AC, Gordon D, Levy D, Lloyd-Jones DM, McBride P, Schwartz JS, Shero ST, Smith SC Jr, Watson K, Wilson PW, Eddleman KM, Jarrett NM, LaBresh K, Nevo L, Wnek J, Anderson JL, Halperin JL, Albert NM, Bozkurt B, Brindis RG, Curtis LH, DeMets D, Hochman JS, Kovacs RJ, Ohman EM, Pressler SJ, Sellke FW, Shen WK, Smith SC Jr, Tomaselli GF; American College of Cardiology/American Heart Association Task Force on Practice Guidelines. 2013 ACC/AHA guideline on the treatment of blood cholesterol to reduce atherosclerotic cardiovascular risk in adults: a report of the American College of Cardiology/ American Heart Association Task Force on Practice
Guidelines. Circulation, 2014; 129 (25 Suppl 2): S1-45

2) Teramoto T, Sasaki J, Ishibashi $S$, Birou $S$, Daida H, Dohi $S$, Egusa $G$, Hiro $T$, Hirobe $K$, Iida $M$, Kihara $S$, Kinoshita M, Maruyama C, Ohta T, Okamura T, Yamashita S, Yokode M, Yokote K; Japan Atherosclerosis Society (JAS). Comprehensive risk management for the prevention of cardiovascular disease: executive summary of the Japan Atherosclerosis Society (JAS) guidelines for the diagnosis and prevention of atherosclerotic cardiovascular diseases in Japan - 2012. J Atheroscler Thromb, 2013; 20: 603-615

3) Catapano AL, Reiner Z, De Backer G, Graham I, Taskinen MR, Wiklund O, Agewall S, Alegria E, Chapman M, Durrington P, Erdine S, Halcox J, Hobbs R, Kjekshus J, Filardi PP, Riccardi G, Storey RF, Wood D; European Society of Cardiology (ESC); European Atherosclerosis Society (EAS). ESC/EAS Guidelines for the management of dyslipidaemias: the Task Force for the management of dyslipidaemias of the European Society of Cardiology (ESC) and the European Atherosclerosis Society (EAS). Atherosclerosis, 2011; 217 Suppl 1: S1-44

4) http://www.uspreventiveservicestaskforce.org/Page/Name/ uspstf-a-and-b-recommendations/

5) Jacobs D, Blackburn $H$, Higgins $M$, Reed D, Iso $H$, McMillan G, Neaton J, Nelson J, Potter J, Rifkind B, Rossouw J, Shekelle R, Yusuf S, DPhil, for Participants in the Conference on Low Cholesterol Mortality Associations Report of the Conference on Low Blood Cholesterol: Mortality Associations. Circulation, 1992; 86: 1046-1060

6) Sugiyama D, Okamura T, Watanabe M, Higashiyama A, Okuda N, Nakamura Y, Hozawa A, Kita Y, Kadota A, Murakami Y, Miyamatsu N, Ohkubo T, Hayakawa T, Miyamoto Y, Miura K, Okayama A, Ueshima H; NIPPON DATA 80/90 Research Group. Risk of hypercholesterolemia for cardiovascular disease and the population attributable fraction in a 24-year Japanese cohort study. J Atheroscler Thromb, 2015; 22: 95-107

7) Daimon M, Oizumi T, Kameda W, Matsui J, Murakami H, Ueno Y, Kubota I, Yamashita H, Kayama T, Kato T. Association of treatment for hyperlipidemia with a decreased total mortality in Japanese individuals: The Yamagata (Takahata) Study. J Atheroscler Thromb, 2015; 22: 1030-1039

8) Hozawa A, Shimazu T, Kuriyama S, Tsuji I. Benefit of home blood pressure measurement after a finding of high blood pressure at a community screening. J Hypertens, 2006; 24: 1265-1271

9) Tanaka T, Okamura T, Yamagata Z, Takebayashi T, Tamura U, Kusaka Y, Urano S, Miyoshi Y, Okayama A, Ueshima H; HIPOP-OHP Research Group.. Awareness and treatment of hypertension and hypercholesterolemia in Japanese workers: the High-risk and Population Strategy for Occupational Health Promotion (HIPOP-OHP) study. Hypertens Res, 2007; 30: 921-928

10) Okamura T, Tanaka H, Miyamatsu N, Hayakawa T, Kadowaki T, Kita Y, Nakamura Y, Okayama A, Ueshima H; NIPPON DATA80 Research Group.. The relationship between serum total cholesterol and all-cause or causespecific mortality in a 17.3-year study of a Japanese cohort. Atherosclerosis, 2007; 190: 216-223 